\title{
Dominant EEG frequencies of patients undergoing dobutamine stress test
}

\author{
A. K. Macpherson ${ }^{1}$, S. Neti ${ }^{1}$, M. Averbach ${ }^{2}$, P. A. Macpherson ${ }^{3}$ \\ \& C. Chutakositkanon ${ }^{1}$ \\ ${ }^{I}$ Institute of Biomedical Engineering and Mathematical Biology, \\ Lehigh University, USA \\ ${ }^{2}$ Division of Cardiology, St Luke's Hospital, USA \\ ${ }^{3}$ Department of Applied Technology, Rogers State University, USA
}

\begin{abstract}
There has been considerable research and speculation that if the brain is under stress then it could affect the heart and lead to heart disease. The purpose of the present research was to examine the inverse problem of whether the heart could potentially cause undesirable reactions in the brain. One method to evaluate for underlying coronary artery disease is to perform stress testing. Often, myocardial stress is achieved by the patient walking on a treadmill while being monitored. In patients who are unable to exercise, pharmacologic stress testing is performed, either with vasodilatory agents (e.g. adenosine) or dobutamine, which is a pro-inotropic and chronotropic drug. During dobutamine infusion, the heart rate increases, but there is a negligible increase in blood pressure. Five patients who were undergoing dobutamine stress testing were instrumented with the standard 19 electrode EEG sensors to record brain activity. It was found that all patients showed resonance in the brain activity at frequencies around $10 \mathrm{~Hz}$. The signal strengths and the electrode locations where a resonance varied between patients. The one location where all of the patients showed resonance was at T5-O1; towards the back of the head and for this location, all patients showed an EEG resonance frequency at approximately $10 \mathrm{~Hz}$. Further analysis of the EEG data is needed to appreciate the consequences of this neurocardilogical phenomenon.
\end{abstract}

Keywords: heart, brain, echocardiograms, neurocardiology, electroencephalographs. 


\section{Introduction}

A review of how the brain regulates the cardiovascular system in given in [1]. A study [2] involved a patient on a bicycle undergoing a stress test that was instrumented with EEG electrodes on the patient's head. The object of the research was to construct a model for determining the effect of stress on patients in an industrial environment. Although only the output of the electrode on the top of the head is reported it is clear that more electrodes were used. The result was a clear indication of a relation between the heart activity and the electroencephalograph (EEG) reading. The regions of the brain which could clearly be identified as independent of blood pressure were the left temporal and the lateral frontal region. The brain activity correlated with the stress induced cardiac activity. The present work is in many ways an extension of that study. There were several aims in the present study. One was to eliminate the possibility that the increase in blood pressure may be the prime cause of any effect observed. A second aim was to use a standard 12 electrode EEG to locate where the signals arose on the brain surface. Finally the longer term objective was to seek relationships between the EEG output and the electrocardiograph (EKG) results.

\section{Method of experimentation and calculation}

In patients that are unable to exercise, pharmacologic stress testing is performed with the vasodilatoryagent dobutamine, which is a pro-inotropic and chronotropic drug. During dobutamine infusion, the heart rate increases, but there is a negligible increase in blood pressure. The result is that the heart rate increases as a function of the dosage. The use of Dobutamine removes the possibility of blood pressure being the cause of the brain activity observed. There were 5 patients involved in the experiments and due to their health state there was a variation in the rate of Dobutamine injected. The highest dosage for a given patient varied dependent on the patient's tolerance and were in the $20 \mathrm{mics} / \mathrm{kg} / \mathrm{sec}$ and $40 \mathrm{mics} / \mathrm{kg} / \mathrm{sec}$ range. Some patients had a maximum of 35 mics. The general aim was to achieve a heart rate of 120 beats per minute.

While the dobutamine was being injected readings from the standard 19 electrodes were recorded. The location of the electrodes is shown in figure 1. A typical EEG output is shown in figure 2.

A significant difference between the present work and that reported in [4] is that in [4] analogue output was used to undertake the calculations whereas in the present work EEG digital data output has been used. This has had an impact on the reliability of the results. Fourier transforms were taken of each of the EEG signals. A very simplified explanation of a Fourier transform [3] which will serve to clarify the concept is as follows. The number the keys on a piano from 1 to 88 and play some music. There is a different frequency and hence different energy of the original overall sound associated with each key. The energy associated with each key is summed over the number of times it is struck during the playing of the music and plotted against the frequency. This is essentially a Fourier transform. 

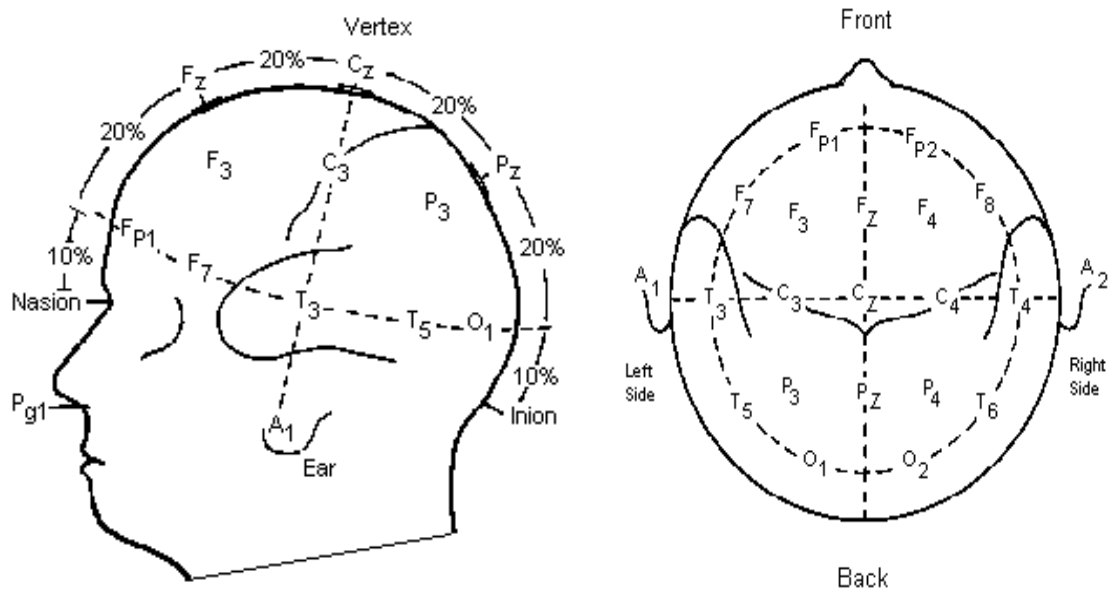

Figure 1: Electrode positions on skull. From web site by Eric Chudler, University of Washington.

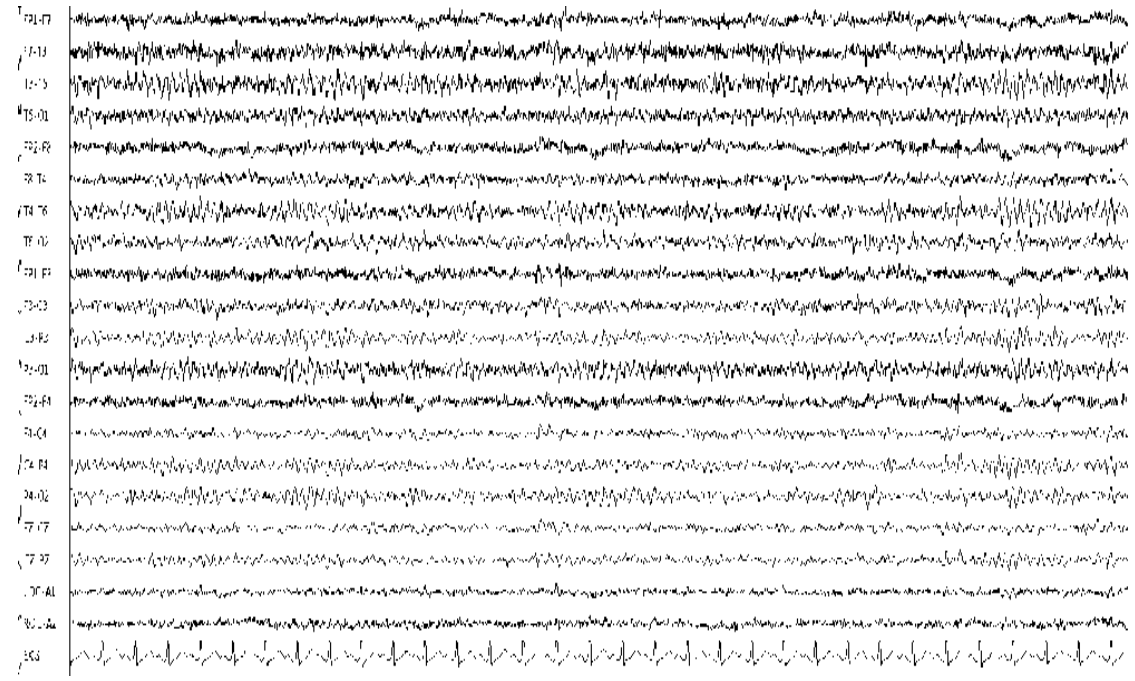

Figure 2: $\quad$ Sample EEG output for a patient.

At the same time as the EEGs were being taken, ECG (EKG) results were also obtained while the dobutamine was administered. The ECG was analyzed using a system known as wavelet analysis. Wavelet analysis shows the variation of various frequency bands with time. The piano analogy may be extended to wavelets. If the piano keys were divided into 5 energy groups for example, 1-20, 21-41, etc., then the wavelet analysis would at any given time account for the total energy for each group and plot it against time. Thus Fourier analysis 
considers for a period of time how much energy is associated for a given frequency whereas wavelet analysis considers the time variation of frequencies.

The results were generated using the MATLAB code.

\section{Results}

Some preliminary results were published in [4] indicating that, using dobutamine to increase heart rate, Fourier transform resonances could be detected in the brain at approximately $10 \mathrm{~Hz}$. The locations where these resonances were found were at the CZ-PZ, C4-P4, T5-O1, T4-T6 and C3-P3 regions. In the present study it was found that resonance was found for EEG data obtained at CZ-PZ, F8-T4, C4-P4, T5-O1, T4-T6, F3-C3, F7-T3, P3-O1, P4-O2, T5-O1, T3-T5, $\mathrm{T} 6 \mathrm{O} 2$ and $\mathrm{C} 3-\mathrm{P} 3$ as designated in Figure 1.

The present study extended earlier work [4] and involved 5 patients. All of the five patients showed varying responses of brain activity. The quality of the transforms varied between different patients as shown in figure 3 .

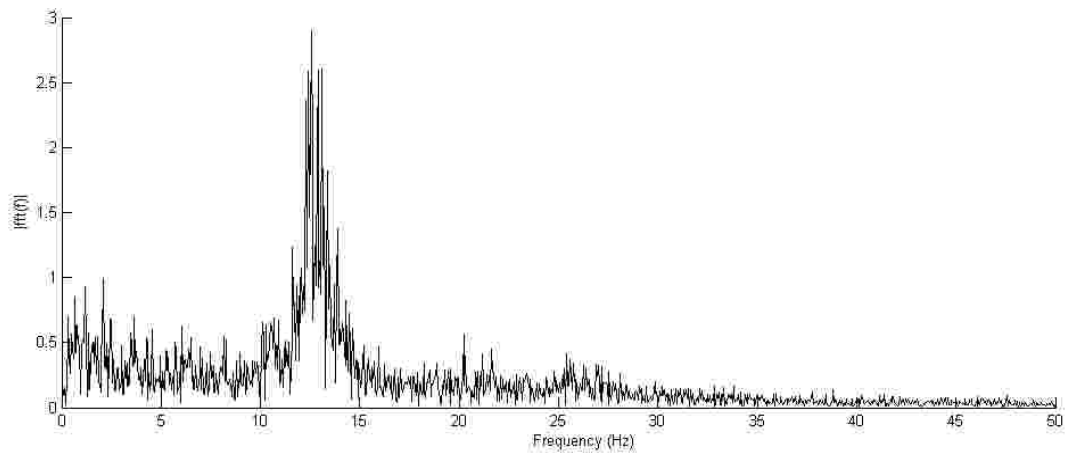

(a)

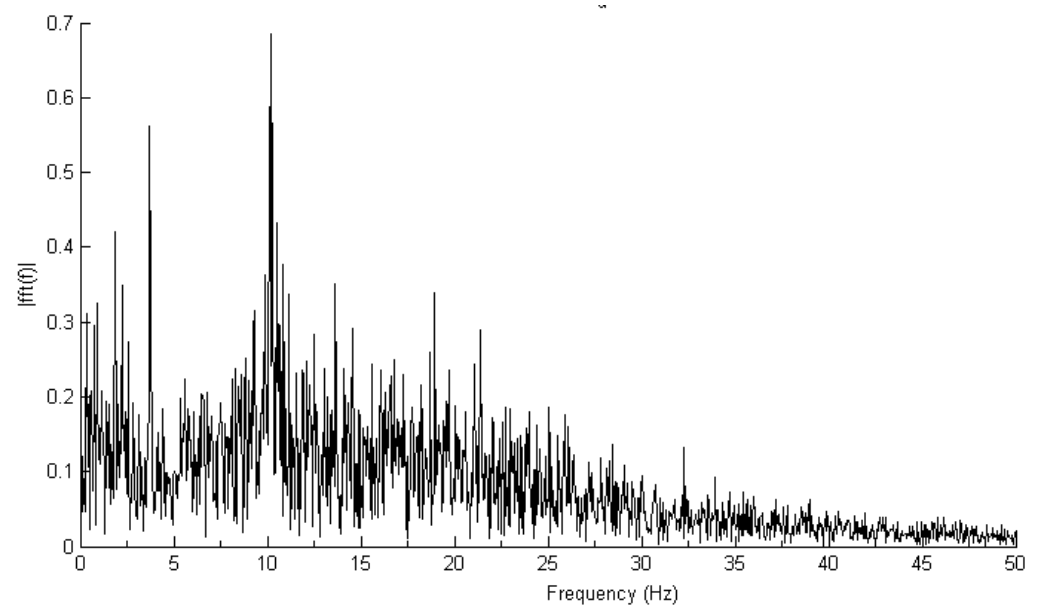

(b)

Figure 3: Variation of Fourier transforms between patients. 
There is a significant variation in the magnitude of the response between patients. The detail shown is due to use of the digital signals from the EEG output. For example heart beats using (EKG) digital data are shown in figure 4.

As it is impossible to discuss all the results here, only the results are shown for patient 3 as representative of the patients who did show resonance at approximately $10 \mathrm{~Hz}$. This patient was chosen as the clinical report on the results of the tests indicated there was no evidence of ischemia and systolic function was normal.

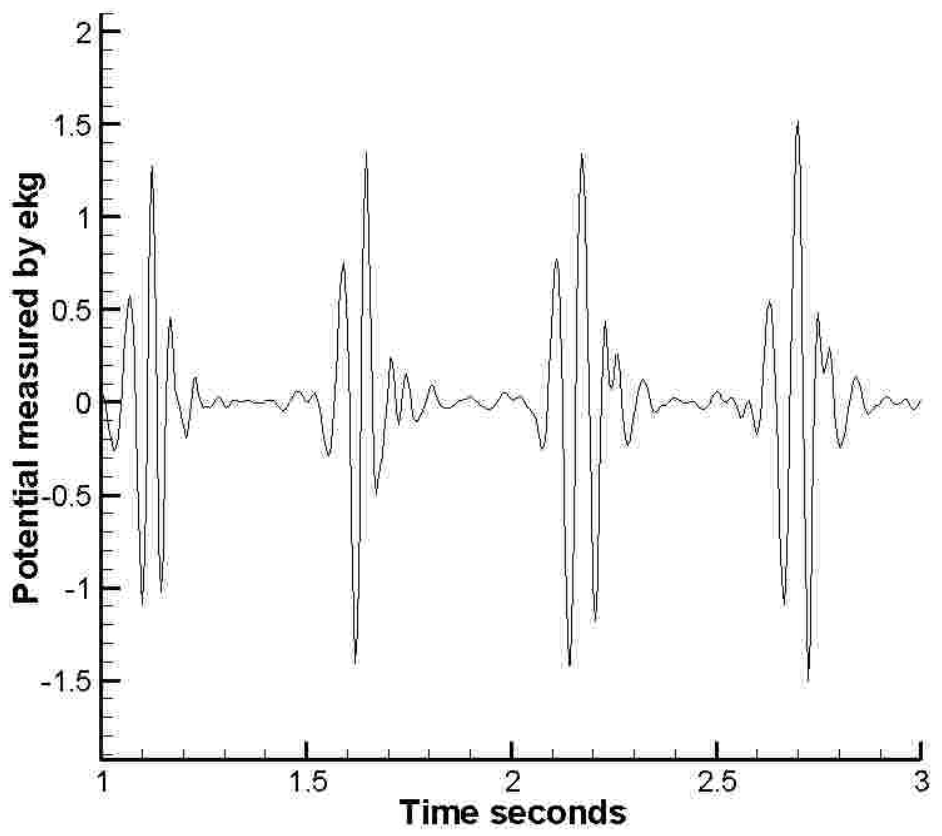

Figure 4: Heart beat from digital signal.

A typical Fourier transform from the EEG results for patient 3 is shown in figure 5.It shows a strong signal at $10 \mathrm{~Hz}$. This persists over the entire time that the heart rate is elevated. When comparing with the rest EEG it is difficult to find extended periods where the patient is calm when undergoing the dobutamine testing.

The results are often similar to figure 3(b) without such a pronounced peak at $10 \mathrm{~Hz}$. There are short periods where there is little brain activity at $10 \mathrm{~Hz}$ but the smallest disturbance will generate signals. For example if the doctor enters the room. This not the case in the elevated heart rate. In addition the heart rate is not constant even at a rest condition. The rest rate of patient 3 was $80 \mathrm{bpm}$. 


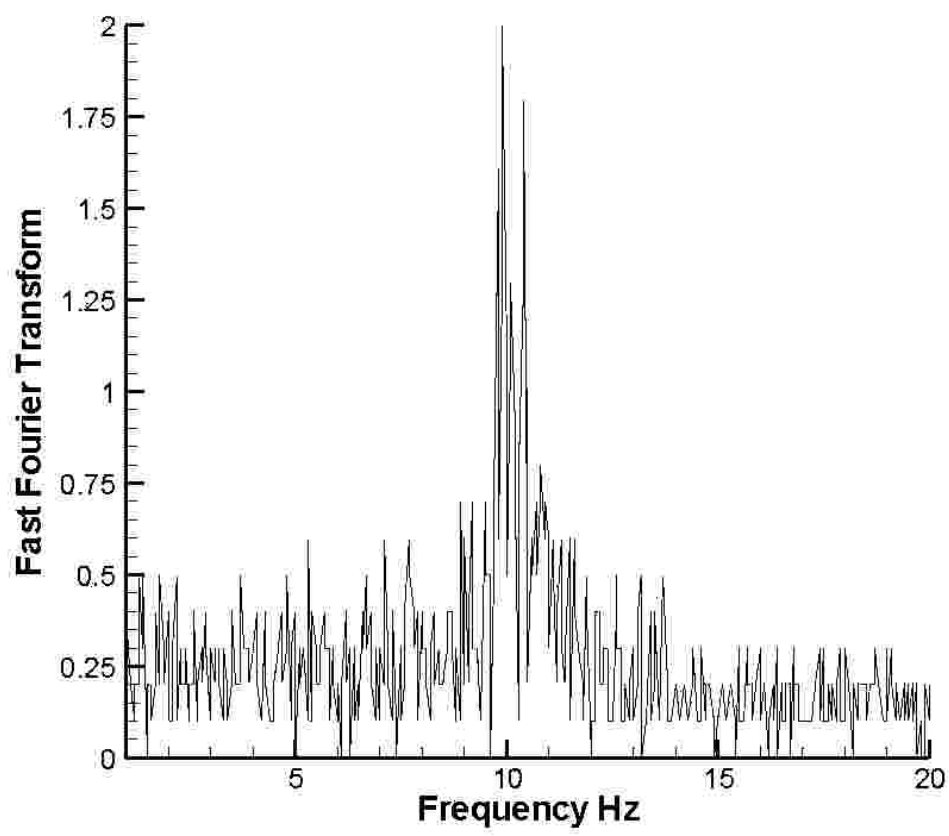

Figure 5: $\quad$ FFT at CZ-PZ.

An investigation was then undertaken to try and determine the cause of these resonances by the heart. Wavelet analysis was undertaken of the heart signals. The results are displayed in bands of different frequencies. The code to generate the wavelets was taken from [5]. A typical result is shown in figure 6.

The upper 4 bands on magnification are similar to figure 4 both at elevated and resting heart rates. The difference occurs in band 5 above. The magnified images of the resting waves is shown in figure 7 and the waves at a heart rate of $120 \mathrm{BPM}$ is shown in figure 8.

It can be seen that there is an increase in frequency at the higher heart rate going from $4 \mathrm{~Hz}$ to $5 \mathrm{~Hz}$. However a significant feature is that at the higher heart rate the signal is better organised. Further research is required to determine if this is the driving mechanism for the resonance in the brain.

\section{Conclusions and future work}

It has been shown that generally resonant frequencies in the brain EEG output appear as the heart rate is increased as part of a dobutamine stress test. The frequency of these resonances is around $10 \mathrm{~Hz}$ for most patients. These resonances could be caused by low frequency waves and harmonics of these waves in the heart beat. 

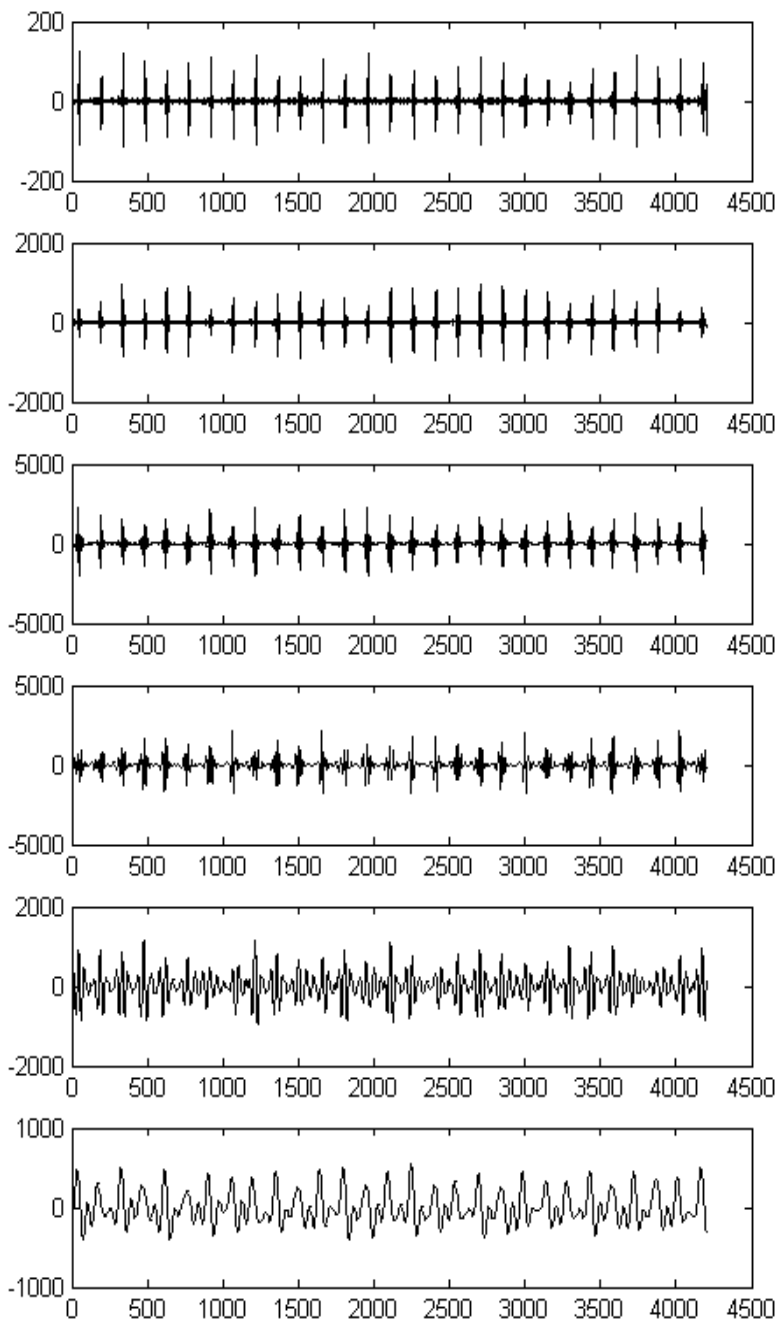

Figure 6: $\quad$ Six frequency bands generated using code from [5]. 


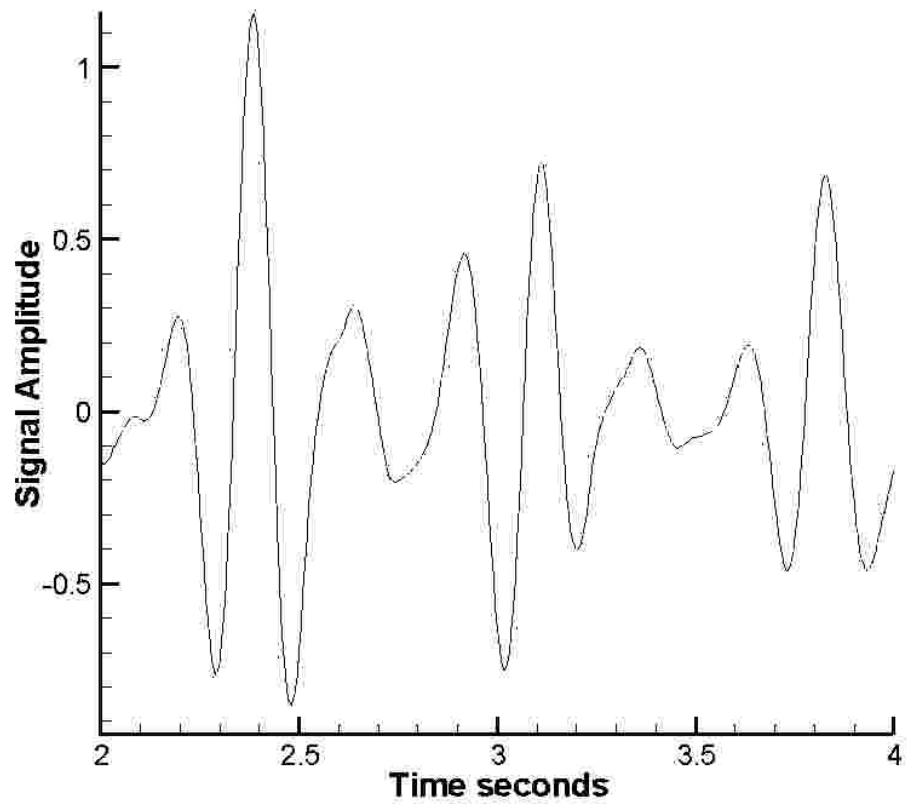

Figure 7: Low frequency wavelet at resting heart rate.

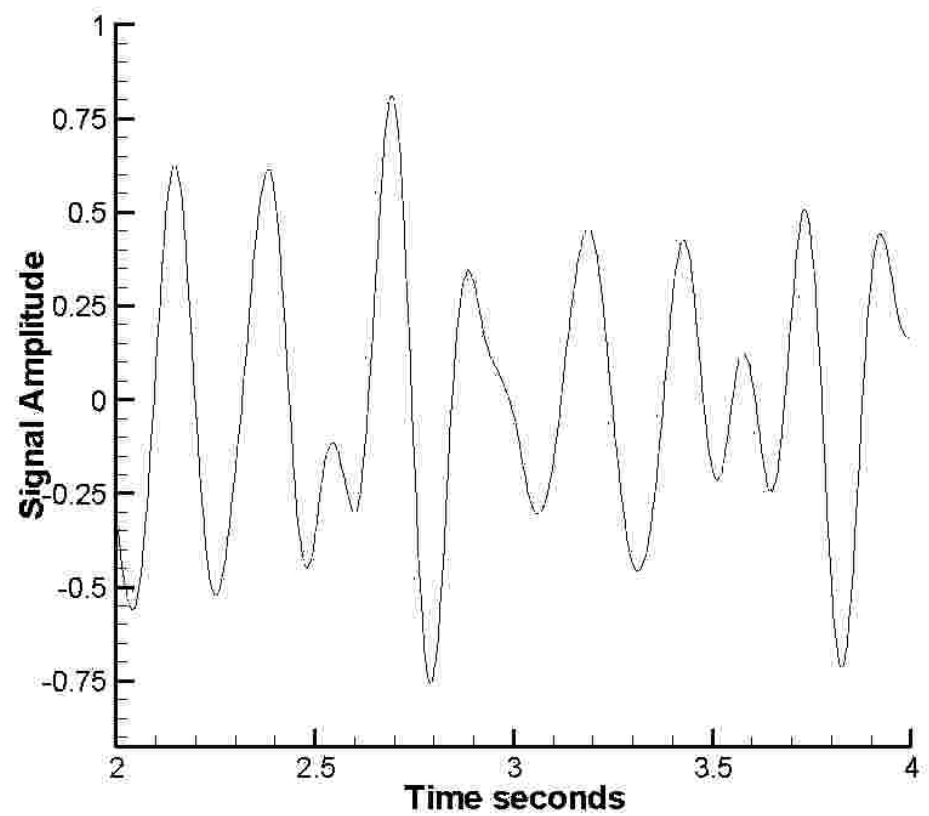

Figure 8: Low frequency wavelet at heart rate $120 \mathrm{bpm}$. 
There may be long term health issues involved with these repeated assaults on the brain due to low frequencies. Besides the possibilities involving disease there is a documented effect (trauma) due to low frequency waves known as wind turbine syndrome. Such trauma including headaches is experienced by people living close to wind turbine farms. Some reports have put that critical frequency at $8 \mathrm{~Hz}$ and the $10 \mathrm{~Hz}$ resonances from the EEG signals reported above are not far from those. Further analyses might bring out the relationship between these two phenomena.

\section{References}

[1] Coote, J.H. Landmarks in understanding the central nervous control of the Cardiovascular System. Experimental Physiology, 92(1), pp 3-18 2007

[2] Ema, El-sd., Mahdi, M., Derick. A.L. A closed-loop hybrid physiological model relating to subjects under physical stress, Artificial Intelligence in Medicine, 38, pp 257-274, 2006.

[3] This analogy is not original to this publication but we are unable to determine the origin of the analogy

[4] Macpherson, A.K., Neti, S., Averbach, M. Macpherson, P.A., Chutakositkanon, C. and Nathanson, D. The Influence of Heart Stress on the Brain: A preliminary Study, Design \& Nature and Ecodynamics, 5(2) 160$172,2010$.

[5] Internet web site M:|uvi_wave|Matlab Uvi_Wave Toolbox.htm 